This manuscript is a preprint and has been submitted for publication in Physical Review E. The manuscript has undergone peer-review but has yet to be formally accepted for publication. Subsequent versions of this manuscript may have slightly different content. 


\title{
Mechanisms controlling fluid break-up and reconnection during two-phase flow in porous media
}

\author{
Catherine Spurin, ${ }^{1}$ Tom Bultreys,${ }^{1,2}$ Branko Bijeljic, ${ }^{1}$ Martin J. Blunt, ${ }^{1}$ and Samuel Krevor ${ }^{1}$ \\ ${ }^{1}$ Department of Earth Science \& Engineering, Imperial College London* \\ ${ }^{2}$ Department of Geology, Ghent University
}

(Dated: September 30, 2019)

\begin{abstract}
The use of Darcy's law to describe steady-state multiphase flow in porous media has been justified by the assumption that the fluids flow in continuously connected pathways. However, a range of complex interface dynamics have been observed during macroscopically steady-state flow, including intermittent pathway flow where flow pathways periodically disconnect and reconnect. The physical mechanisms controlling this behaviour have remained unclear, leading to uncertainty concerning the occurrence of the different flow regimes. We observe that the fraction of intermittent flow pathways is dependent on the capillary number and viscosity ratio. We propose a phase diagram within this parameter space to quantify the degree of intermittent flow.
\end{abstract}


Flow through porous media in the Earth's subsurface is central to many societal challenges including the geological storage of carbon dioxide, hydrocarbon recovery and aquifer contamination containment. Typically, macroscopic flow behaviour in porous media is described using Darcy's law extended to multiphase flow. This is a continuum mechanical description, in which the constitutive properties are the relative permeability and capillary pressure [1-3]. This relies on the longstanding assumption that the interface between fluid phases is invariant at a fixed saturation, such that once a pathway is established, it will remain stable; this is connected pathway flow [4-6].

However, it has been observed that a stable partitioning of fluids in the pore space does not always occur - with interfaces between the fluid phases able to dynamically rearrange, even during steady-state flow where the average fluid saturations are invariant $[7,8]$. A number of studies have observed the advection of discrete clusters of non-wetting phase fluid through the pore space. These clusters are referred to as ganglia and the phenomenon is broadly described as ganglion dynamics $[9,10]$. This has been observed to occur at high fluid flow rates, where viscous forces dominate over capillary forces [8, 10-13]. Increasing the viscous force results in increased breakup and ganglia advection. This process is well understood and of less relevance to the subsurface, where flow rates are normally too low for such behaviour to occur.

Fluid displacements with complex interface dynamics have also been observed in flow regimes dominated by capillary forces. A number of groups have now observed that flow in porous rocks can occur along pathways that periodically disconnect and reconnect in a series of snap-off and reconnection events [4,14-17]. This is akin to the operation of traffic lights, with critical pathways opening and closing, instead of discrete ganglia moving through a system, and is referred to as intermittent pathway flow. Its presence in subsurface flow will impact the energy dissipation of the fluids. Intermittency has been observed to relate to the balance of capillary and viscous forces, defined by a dimensionless capillary number [14]. However, at similar capillary numbers, fluid systems with more viscous non-wetting phase have less intermittency [15]. It has been hypothesized that intermittency may also depend on inertial forces [18].

There remain uncertainties around the circumstances under which a given flow behaviour - ganglion dynamics, intermittency, or connected pathway flow - will prevail, and what force balance controls these deviations from connected pathway flow. In this work, we evaluate 
how this behaviour is influenced by the balance between capillary, viscous, and inertial forces. We characterise the role of controlling system parameters through a number of experiments across a range of fluid flow rates, and with different fluid pairings.

Capillary forces dominate at the pore scale in subsurface flows. So often a capillary number, which is the ratio of viscous forces to capillary forces, is used to describe a flow regime. The most widely used capillary number is defined as:

$$
C a=\frac{q \mu}{\sigma}
$$

where $q$ is the flow per unit area, $\sigma$ is the interfacial tension and $\mu$ is the viscosity. This results in a capillary number specific to each fluid phase present in the system.

We define a capillary number, $C a_{t}$, which characterises the whole system, rather than each individual phase. Assuming that capillary pressure is spatially invariant at steadystate, the total flow per unit area, $q_{t}$, will be proportional to the permeability of the porous medium, $K$, the total mobility of the fluids, $\lambda_{t}$, and the pressure gradient from viscous dissipation, $\nabla P_{v}[19]$ :

$$
q_{t}=-K \lambda_{t} \nabla P_{v}
$$

In one dimension, this can be rearranged so that a typical pressure drop over a length $L$ can be written as:

$$
\Delta P_{v}=\frac{q_{t} L}{K \lambda_{t}}
$$

The total mobility, $\lambda_{t}$, is defined as the ratio of the relative permeability to phase viscosity:

$$
\lambda_{t}=\frac{k_{r, n w}}{\mu_{n w}}+\frac{k_{r, w}}{\mu_{w}}
$$

where $k_{r}$ is relative permeability [5].

The Young-Laplace equation describes the capillary pressure, $P_{c}$, as a function of the interfacial tension and the average pore size, $r$ [5]:

$$
P_{c} \propto \frac{\sigma}{r}
$$

The ratio of $\Delta P_{v}$ to $P_{c}$ results in:

$$
\frac{r L}{K} \frac{q_{t}}{\sigma \lambda_{t}}=\frac{r L}{K} C a_{t}
$$

thus,

$$
C a_{t}=\frac{q_{t}}{\sigma \lambda_{t}}
$$


To avoid the inclusion of relative permeability, which is rock dependent, it is beneficial to rewrite Equation 4 to include the fractional flow, which is imposed in our experiments. The fractional flow is the fraction of the flow rate constituted by the wetting fluid flow $q_{w}$ :

$$
f_{w}=\frac{q_{w}}{q_{t}} .
$$

Approximating $k_{r, w} \sim f_{w}$ and $k_{r, n w} \sim 1-f_{w}$ the capillary number can be written as:

$$
C a_{t}=\frac{q_{t}}{\sigma\left(\frac{\left(1-f_{w}\right)}{\mu_{n w}}+\frac{f_{w}}{\mu_{w}}\right)} .
$$

If intermittent pathway flow is controlled purely by the capillary number, an increase in flow rate and an increase in the viscosity of the fluids will both result in an increase in the amount of intermittency. However, as we show later, the viscosity ratio, $M$, of the fluids:

$$
M=\frac{\mu_{n w}}{\mu_{w}}
$$

also affects the behaviour. When $M<1$ and at high flow rates, the displacement of the wetting phase by the non-wetting phase is unstable, resulting in a ramified, finger-like percolation pattern [20,21]. This pattern is more prone to snap-off of the non-wetting phase by the wetting phase. We hypothesise that the role of the viscosity ratio is important even at low capillary numbers, and that when the non-wetting phase has a lower viscosity than the wetting phase $(M<1)$, more intermittency can occur for the same capillary number because the non-wetting phase can easily be disconnected by snap-off.

If intermittent pathway flow is controlled by inertial forces, then non-wetting phases with similar densities will exhibit similar flow regimes, for a given flow rate. By varying the densities and viscosities of the fluids, we can evaluate the impact of inertial forces, which were not observed to play a significant role.

We performed a series of two-phase, steady-state co-injection experiments in a laboratory based micro-CT scanner for a cylindrical Estaillades carbonate rock sample $6 \mathrm{~mm}$ in diameter and $21 \mathrm{~mm}$ in length. The sample was initially saturated with brine and scanned, this is the brine-saturated scan. Then the non-wetting phase, which was either nitrogen, decane, or hexadecane, was injected simultaneously with the brine, with constant back pressure. The fractional flow, $f_{w}$, was varied between experiments, decreasing in a drainage sequence while the total volumetric flow rate was kept constant. Steady-state was achieved before taking a scan. Steady-state was determined by the stabilisation of the differential pressure measured 
TABLE I: Fluid parameters for nitrogen, decane, hexadecane and brine. Density values for decane and hexadecane from VWR International. Viscosity and interfacial tension for decane from [15], and for hexadecane from [22] and [23]. Remaining values from PubChem, open chemistry database.

\begin{tabular}{|l|l|l|l|}
\hline Fluid & $\begin{array}{c}\text { Viscosity } \mu \\
(\mathbf{P a} \cdot \mathbf{s})\end{array}$ & $\begin{array}{c}\text { Density } \rho \\
\left(\mathbf{k g} / \mathbf{m}^{3}\right)\end{array}$ & $\begin{array}{l}\text { Interfacial tension } \\
\text { with brine } \sigma(\mathbf{N} / \mathbf{m})\end{array}$ \\
\hline Nitrogen & $2.08 \times 10^{-5}$ & 110 & $64 \times 10^{-3}$ \\
\hline Decane & $83.8 \times 10^{-5}$ & 730 & $47 \times 10^{-3}$ \\
\hline Hexadecane & $34.5 \times 10^{-4}$ & 773 & $53 \times 10^{-3}$ \\
\hline Brine & $82.0 \times 10^{-5}$ & 1263 & $\mathrm{~N} / \mathrm{A}$ \\
\hline
\end{tabular}

across the sample $[7,8,15]$. Injection of the fluids continued during a scan. A more detailed description of the experimental procedure is given in [? ].

There are four sets of observations described in this work:

1. Nitrogen/brine injection at $0.8 \mathrm{ml} / \mathrm{min}$ for $f_{w}=1,0.85,0.7,0.5,0.25,0$. The observations from this experiment are referred to as the high $\mathrm{Ca}$ nitrogen observations.

2. Nitrogen/brine injection at $0.1 \mathrm{ml} / \mathrm{min}$ for $f_{w}=1,0.85,0.7,0.5,0.25,0$. These are referred to as the low $C a$ nitrogen observations.

3. Decane/brine injection at $0.02 \mathrm{ml} / \min$ for $f_{w}=1,0.85,0.7,0.5,0.25,0$. The capillary number for the non-wetting phase is matched to the high $\mathrm{Ca}$ nitrogen experiment. These are referred to as the decane observations.

4. Hexadecane/brine injection at $0.02 \mathrm{ml} / \mathrm{min}$ for $f_{w}=1,0.85,0.7,0.5,0.25,0$. These are referred to as the hexadecane observations.

Their location within the parameter space defined by the capillary number (Equation 9) and viscosity ratio (Equation 10) is shown in Figure 1 alongside previous experiments $[4,14,15$, 17]. The properties of the fluids are given in Table I.

The X-ray images were acquired over a period of 40 minutes. This acquisition time was necessary for a sufficient X-ray count to be collected by the flat panel detector for the projections to be reconstructed with minimal artefacts in the final greyscale images. This 


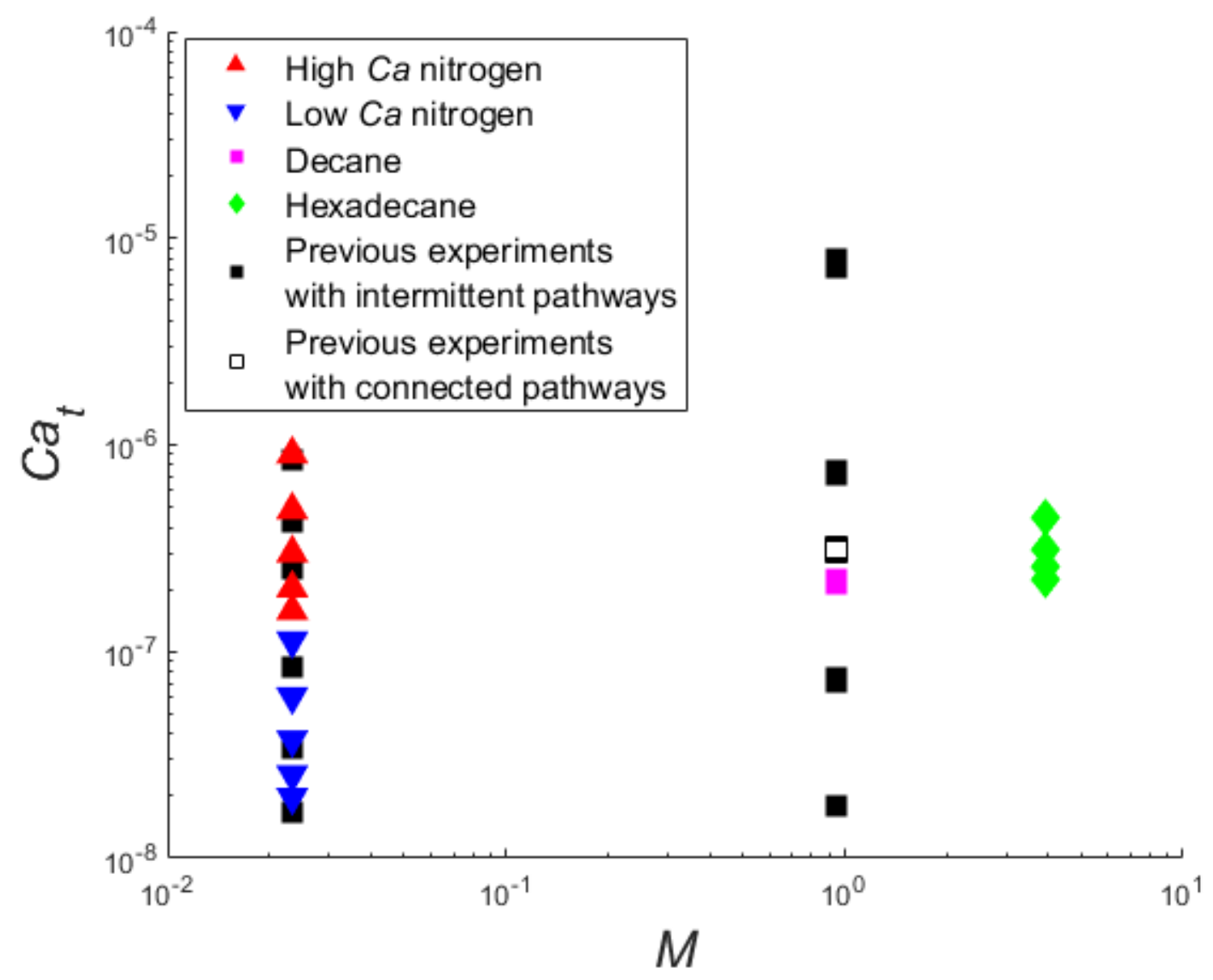

FIG. 1: Capillary numbers and viscosity ratios explored in this research (colour), compared to observations from the literature for a sandstone (black) $[4,14,15,17]$.

means that intermittent connections cannot be directly observed, and have to be inferred from time-averaged images.

A technique was developed, building on [24] and [15], to identify locations of intermittency in the time averaged imagery. The greyscale value recorded for a voxel within an image depends on the average atomic number of the atoms and the density within the voxel. If there was no movement during a scan, only two greyscale values would be identifiable in the pore space: one for the non-wetting phase, and one for brine. If both brine and the non-wetting phase occupy a pore at some point during a scan, then the resulting image will have a greyscale value between the two phases, dependent on the amount of time each phase was occupying that pore, resulting in an intermediate greyscale value, as shown in Figure 2a. 
Differential imaging was used between the brine-saturated scan and the fractional flow scan in question to find volumes that contain either the non-wetting phase or the intermediate greyscale. Then a simple greyscale threshold was used to segment the intermediate greyscale value from the non-wetting phase (Figure $2 \mathrm{~b}$ ). Partial volume effects are an artefact of the finite resolution of the images; they are 1 voxel thick and occupy the interface between the phases. These were removed from the intermittency segmentation by eroding the segmented intermediate greyscale by 1 voxel and then dilating the result by 1 voxel. Figure 2c shows the final segmentation of the volumes with intermittent pathway flow.

a)

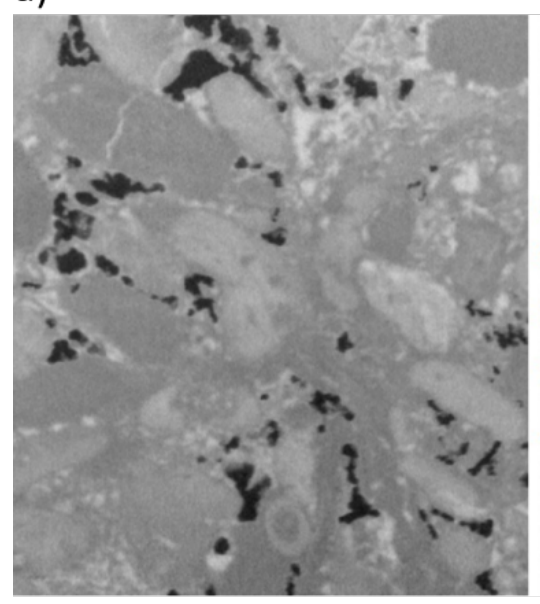

b)

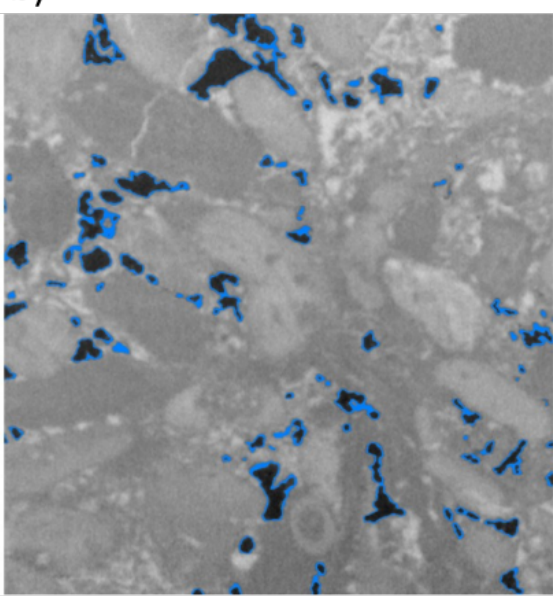

c)

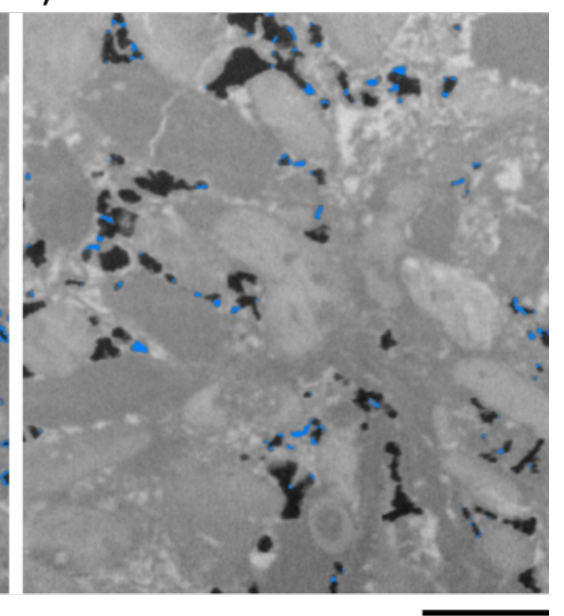

$1 \mathrm{~mm}$

FIG. 2: a) Greyscale data taken during the co-injection of nitrogen and brine for the $f_{w}=$ 0.5 high $\mathrm{Ca}$ nitrogen scan, b) segmentation of the intermediate greyscale shown in blue for the same scan as a), and c) segmentation of the intermittent phase shown in blue for the same scan as a). Flow is out of the page.

For a given viscosity ratio, the capillary number plays an important role in the generation of intermittency. This is shown for the nitrogen experiments in Figure 3, where more intermittency was observed at higher $C a_{t}$. At similar capillary numbers, the amount of intermittency decreases as the viscosity of the displacing non-wetting phase increases, as shown in Figure 3.

A comparison of the decane and hexadecane experiments provides insight into the impact of the viscosity ratio on the generation of intermittency. The flow rates for the experiments are identical and the density ratios are comparable ( 0.58 for decane/brine and 0.61 for hex- 


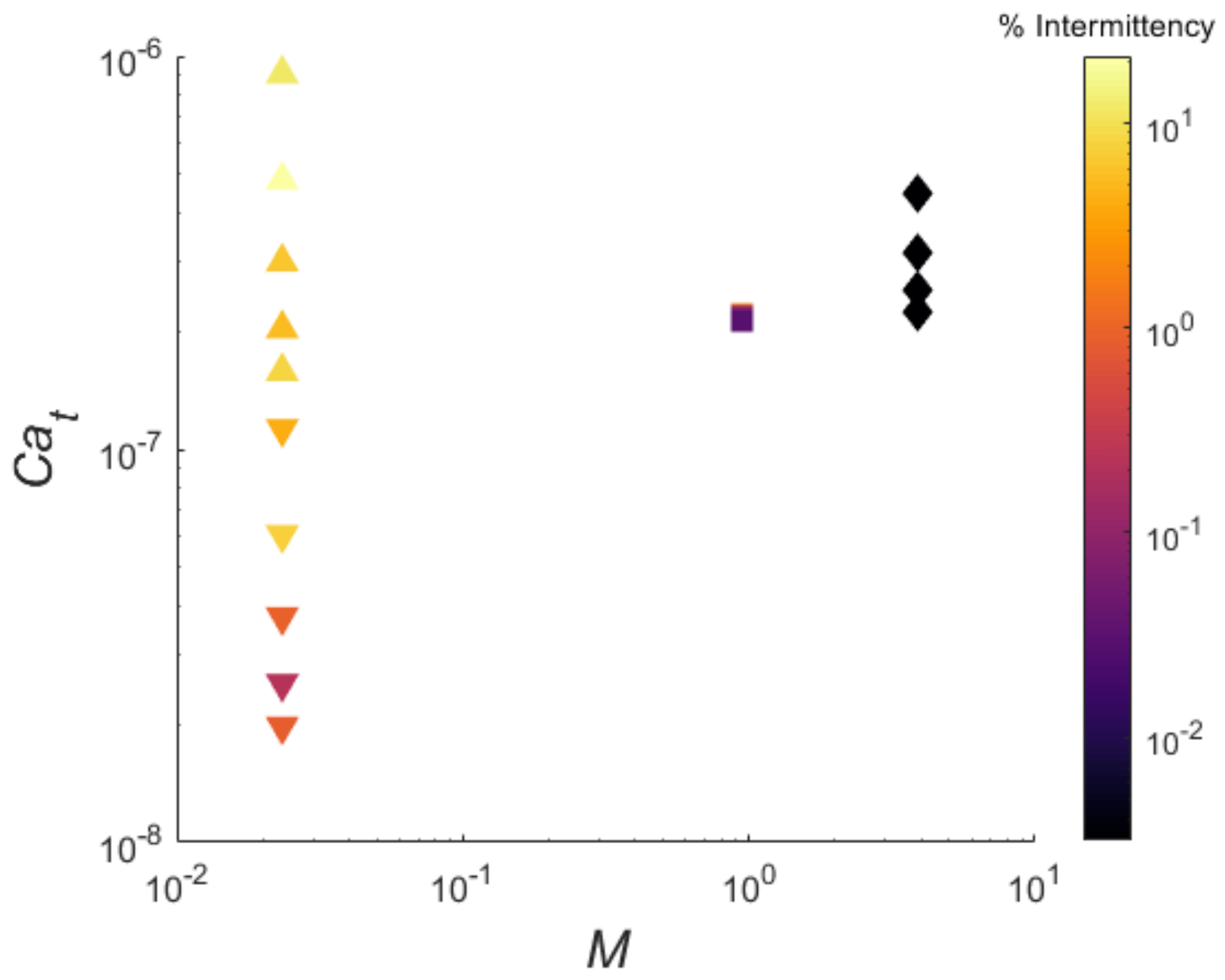

FIG. 3: Viscosity ratio against the capillary number for the total flow, colour coded to the amount of intermittency identified in the system. The \% intermittency is the percentage volume of the pore space identified as containing an intermittent pathway during a scan.

adecane/brine), but the viscosity ratio are distinct ( $M=1.02$ for the decane experiment and $M=4.15$ for the hexadecane experiment). This means that the inertial forces are comparable, but the viscous forces are not. The hexadecane experiments showed no intermittent pathway flow, such that a more viscous non-wetting phase stabilises the flow and allows connected pathway flow [21]. By increasing the viscosity of the non-wetting phase, we increase the stability of the immiscible displacement. A more stable displacement pattern means the non-wetting phase will have a less ramified structure, and less branching fingers of the non-wetting phase that would be more prone to brine reinvasion.

It is observed in this work that intermittency is controlled by the ratio of viscous forces to 
capillary forces (the capillary number) and the viscosity ratio. We, therefore, propose that steady-state two-phase flow dynamics can be classified with a phase diagram in the capillary number and viscosity ratio parameter space (Figure 4). This is similar to the drainage phase diagram first proposed by Lenormand et al. [21] and suggests a link between network-scale fluid morphology and the occurrence of intermittency. Within capillary dominated regimes, intermittent pathways are favourable at low viscosity ratios. At higher viscosity ratios, connected pathway flow is more favourable and intermittent pathway flow may never exist, or is only possible at extremely high flow rates. The precise location of the transitions between intermittent pathway flow, ganglion dynamics and connected pathway flow in Figure 4 are speculative and requires further research. Intermittent pathways can coexist alongside stable pathways so it is likely the transition will be gradual [17].

The transition of intermittent pathways to discrete ganglia advecting through the system either independently or through coalescence and break up is a topic that requires further study. More intermittency was observed for the same fluid pairings and flow rate in our work on a more heterogeneous carbonate than previous work in a more homogeneous sandstone [15]. This implies more rarefied patterns emerging from the pore structure also controls the stability of interfaces, but to a lesser degree than the viscosity ratio. Thus the pore geometry may influence the boundaries in Figure 4; this is an area of future research. At the lowest $C a_{t}$ observed in this work, the volumes identified as intermittent are less than $5 \%$ of the total pore space. This suggests that connected pathway flow may be possible for $M<1$ for values of $C a_{t}<10^{-8}$, but this would require further experiments.

Indicative values of capillary number and viscosity ratio for engineered subsurface processes are shown in Figure 4. Intermittent pathway flows are relevant for geological carbon storage, and the production of natural gas. They should therefore be considered in modelling attempts as intermittent pathways impact larger scale flow properties [? ]. Conventional oil production may be controlled by connected or intermittent pathway flow depending on the setting. Connected pathway flow is expected in heavy oil production, even with the addition of polymers.

We conclude that intermittency is dependent on the capillary number and viscosity ratio. For $M<1$ we observed intermittency at low capillary numbers, but connected pathway exists when $M>1$. The similarity in parameter space between occurrences of intermittency and larger scale flow patterns suggests a link between network-scale fluid structure and the 


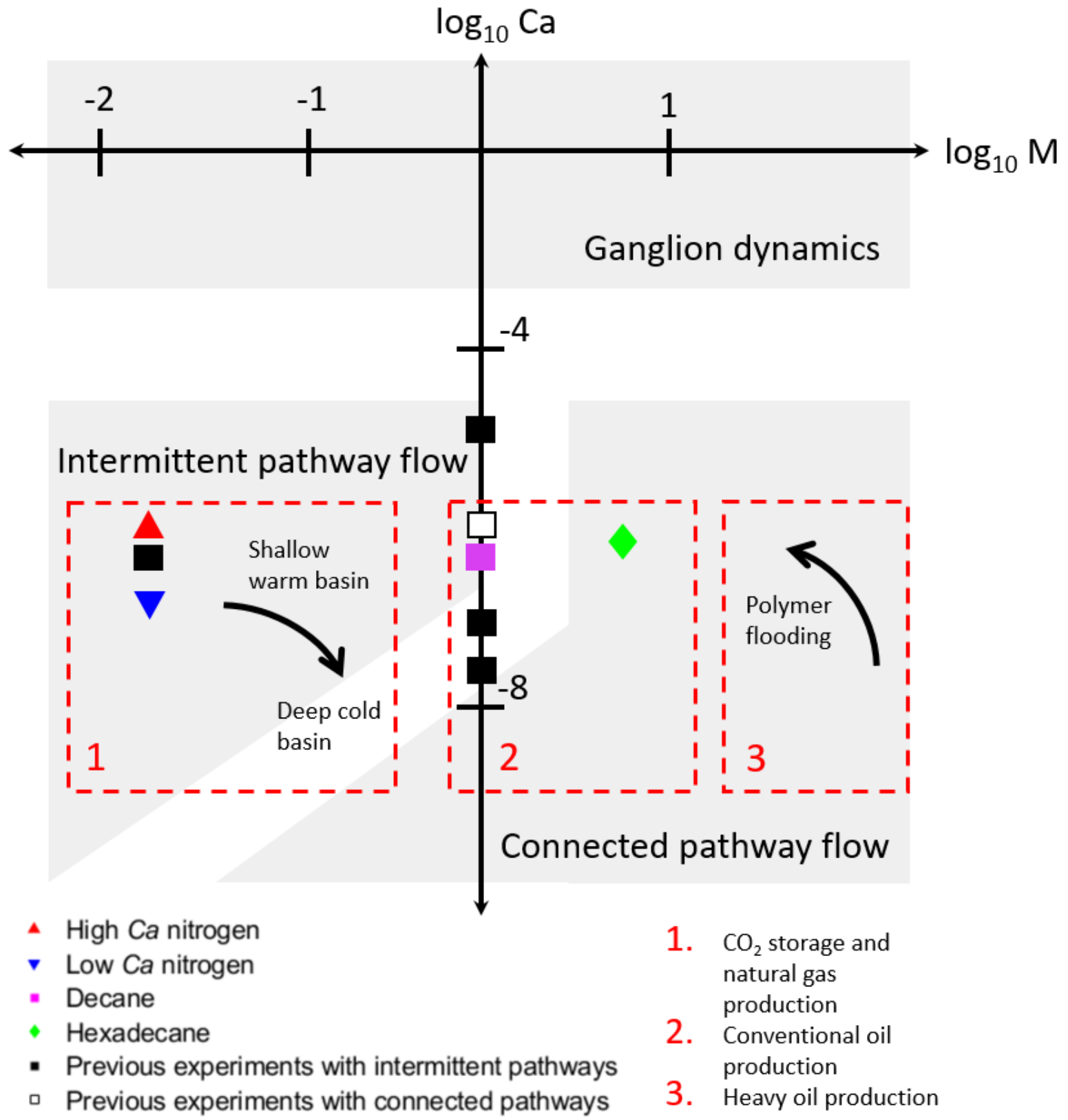

FIG. 4: Phase diagram for steady-state two-phase flow with average experimental results plotted. White areas denote transition zones between the different steady-state flow regimes, although it should be noted that these boundaries are speculative and require further research to be quantified. Red dashed boxes denote the areas relevant to certain subsurface scenarios, highlighting the importance of this work to environmental and industrial applications, with typically capillary numbers taken from [5], viscosity ratios for gaseous systems from [25], viscosity ratios for oils from [26], and the impact of polymer flooding from [27] and [28]. 
occurrence of intermittency [21]. Future work using fast tomography will allow the imaging of intermittent pathways at a range of viscosity ratios and provide a deeper understanding into the dynamics of intermittent pathway flow, and the transitions between the flow regimes.

\section{ACKNOWLEDGMENTS}

A special thanks to Steffen Berg, Alessio Scanziani and Gaetano Garfi for providing interesting debates and to Ying Gao, Hannah Menke and Kamaljit Singh for their support during the experiments. Catherine Spurin is grateful for her funding through the President's PhD Scholarship, Imperial College London. The Shell Digital Rocks Programme is thanked for funding the experimental work. We are also grateful to all our colleagues within this programme for their useful discussions and support. Tom Bultreys acknowledges the Research Foundation-Flanders (FWO) for his post-doctoral fellowship 12X0919N.

* cls13@ic.ac.uk

[1] R. Juanes, E. Spiteri, F. Orr, and M. Blunt, Water Resources Research 42 (2006)

[2] J. Niessner, S. Berg, and S. M. Hassanizadeh, Transport in Porous Media 88, 133 (2011)

[3] M. Muskat, The Physical Principles of Oil Production (McGraw-Hill Book Company, 1949)

[4] R. T. Armstrong, J. E. McClure, M. A. Berrill, M. Rücker, S. Schlüter, and S. Berg, Physical Review E 94, 043113 (2016)

[5] M. J. Blunt, Multiphase Flow in Permeable Media: A Pore-Scale Perspective (Cambridge University Press, 2017)

[6] F. A. Dullien, Porous media: fluid transport and pore structure (Academic Press, 1992)

[7] K. T. Tallakstad, H. A. Knudsen, T. Ramstad, G. Løvoll, K. J. Måløy, R. Toussaint, and E. G. Flekkøy, Physical Review Letters 102, 074502 (2009)

[8] K. T. Tallakstad, G. Løvoll, H. A. Knudsen, T. Ramstad, E. G. Flekkøy, and K. J. Måløy, Physical Review E 80, 036308 (2009)

[9] D. Avraam and A. Payatakes, Journal of Fluid Mechanics 293, 207 (1995)

[10] S. S. Datta, T. Ramakrishnan, and D. A. Weitz, Physics of Fluids 26, 022002 (2014)

[11] A. Gupta and R. Kumar, Physics of Fluids 22, 122001 (2010) 
[12] T. Ramstad and A. Hansen, Physical Review E 78, 035302 (2008)

[13] H. Liu and Y. Zhang, Journal of Applied Physics 106, 034906 (2009)

[14] C. A. Reynolds, H. Menke, M. Andrew, M. J. Blunt, and S. Krevor, Proceedings of the National Academy of Sciences 114, 8187 (2017)

[15] Y. Gao, Q. Lin, B. Bijeljic, and M. J. Blunt, Water Resources Research 53, 10274 (2017)

[16] M. Rücker, W.-B. Bartels, K. Singh, N. Brussee, A. Coorn, H. van der Linde, A. Bonnin, H. Ott, S. Hassanizadeh, M. Blunt, et al., Geophysical Research Letters (2019)

[17] M. Rücker, S. Berg, R. Armstrong, A. Georgiadis, H. Ott, A. Schwing, R. Neiteler, N. Brussee, A. Makurat, L. Leu, et al., Geophysical Research Letters 42, 3888 (2015)

[18] A. Ferrari and I. Lunati, Advances in water resources 74, 1 (2014)

[19] M. Muskat, Soil Science 46, 169 (1938)

[20] K. J. Måløy, J. Feder, and T. Jøssang, Physical Review Letters 55, 2688 (1985)

[21] R. Lenormand, E. Touboul, and C. Zarcone, Journal of Fluid Mechanics 189, 165 (1988)

[22] R. C. Hardy, Journal of Research of the National Bureau of Standards 61, 433 (1958)

[23] A. Goebel and K. Lunkenheimer, Langmuir 13, 369 (1997)

[24] Q. Lin, Y. Al-Khulaifi, M. J. Blunt, and B. Bijeljic, Advances in Water Resources 96, 306 (2016)

[25] J. M. Nordbotten, M. A. Celia, and S. Bachu, Transport in Porous media 58, 339 (2005)

[26] A. Mai, A. Kantzas, et al., Journal of Canadian Petroleum Technology 48, 42 (2009)

[27] O. Y. Hsu and N. S. Hsu, "Alkaline surfactant polymer flooding composition and process," (2000), US Patent 6,022,834

[28] A. Skauge, N. Zamani, J. Gausdal Jacobsen, B. Shaker Shiran, B. Al-Shakry, and T. Skauge, Colloids and Interfaces 2, 27 (2018) 